\title{
Eyelid Tumors: Clinical Data from an Eye Center in Ankara, Turkey
}

\author{
Fatih Cakir Gundogan ${ }^{1 *}$, Umit Yolcu ${ }^{1}$, Ahmet Tas' ${ }^{1}$, Omer Faruk Sahin ${ }^{2}$, Salih \\ Uzun $^{2}$, Hakan Cermik ${ }^{3}$, Sukru Ozaydın ${ }^{3}$, Abdullah Ilhan ${ }^{1}$, Salih Altun ${ }^{1}$, Mustafa \\ Ozturk $^{4}$, Fahri Sahin ${ }^{5}$, Uzeyir Erdem ${ }^{5}$
}

\begin{abstract}
Introduction: The aim of the study was to explore the distribution of eyelid tumors in Ankara, the capital city of Turkey, from a histopathological point of view. Materials and Methods: Medical records of 1,502 patients who had eyelid surgery because of tumoral lesions were retrospectively reviewed after obtaining institutional review board approval. A total of 1,541 lesions with histopathologic diagnosis were included. Inflammatory tumoral lesions were excluded. The lesions were categorized into three groups according to the origin: epidermal, adnexal tumors and 'others', including melanocytic, neural and vascular lesions. Results: Of the total of 1,541, 908 lesions were epidermal in origin. Only $22(1.5 \%)$ were malignant, and $6.0 \%$ was premalignant lesions such as actinic keratosis and Bowen's disease. Twenty-one of 22 malignant lesions were basal cell carcinoma. There was only one patient with squamous cell carcinoma and no sebaceous cell carcinoma. Among the benign tumors $(\mathbf{9 2 . 5 \%})$, squamous papilloma was the most frequent $(\mathbf{2 1 . 8 \%}$ of all lesions). The other frequent lesions were nevus $(\mathbf{1 7 . 6 \%})$, seborrheic keratosis $(\mathbf{1 7 . 3 \%})$, hydrocystomas $(\mathbf{1 0 . 6 \%})$, xanthelasma $(\mathbf{7 . 6 \%})$ and epidermal cysts $(\mathbf{7 . 2 \%})$. Conclusions: The results of this study are in accordance with published literature. The absence of sebaceous cell carcinomas needs to be stressed.
\end{abstract}

Keywords: Eyelid tumors - benign eyelid tumors - eyelid malignancy

Asian Pac J Cancer Prev, 16 (10), 4265-4269

\section{Introduction}

Eyelid tumors are commonly diagnosed neoplasms in routine ophthalmology practice. Some malignant tumors may mimic benign neoplastic and, inflammatory conditions and cause late diagnosis. A wide variety of benign and malignant tumors can originate because of the existence of many different tissue types in the eyelids (Deprez and Uffer, 2009). Different cell types of epidermis, Meibomian, Zeis, Moll, Wolfring and Krause glands, eyelash follicles may all cause eyelid tumors. Eyelid lesions represent $15 \%$ of face tumors and 5-10\% from all cutaneous tumors (Deprez and Uffer, 2009). The maximum incidence is after 60-year-old, and most tumors are situated in the lower eyelid and internal canthus (Salomon et al., 2004; Coroi et al., 2010). Some studies emphasized the importance of sun exposure in the emergence and the use of glasses in the prevention of cancers including the eye and eyelids.(Bhurgri et al., 2003; Turgay et al., 2005; Gajda and Kaminska-Winciorek, 2014) Although many types of eyelid tumors may easily be diagnosed from a clinical point of view, some should be diagnosed with pathological specimens. Only a small number of large studies exist exploring the frequency of eyelid lesions from different sites of the world (Abdi et al., 1996; Ni, 1996; Xu et al., 2008).

This study aimed to explore the distribution of eyelid tumors as diagnosed by histopathologic specimens in Sincan region of Ankara, the capital city of Turkey.

\section{Materials and Methods}

This retrospective study was performed in private eye center, which is located in Sincan, Ankara, the capital city of Turkey. Almost five hundred thousand citizens live in sincan. All patients who had eyelid surgery because of tumoral lesions between October 2008 and March 2012 were retrospectively reviewed. Patients who did not have histopathology report were excluded from the study. The tumors were classified based on origins such as tumors of the epidermis/dermis, tumors of melanocytic origin, and those of glandular, neural, vascular, metastatic, xanthomatous, histiocytic, and inflammatory origin. However, the last group (inflammatory lesions) such as chalazia were not reported in this study. The method for the classification of eyelid tumors was performed by using a previous large study by Deprez $\mathrm{M}$ et al. in order to be able to make a comparison (Deprez and Uffer, 
2009). In that study, the authors used (1)1994 edition of the AFIP atlas of tumor pathology with reference to the revised 2006 edition and from three other sources about eyelid tumors. According to this classification, eyelid tumors were grouped into three main categories. These categories are (1) Epidermal tumors, (2) Adnexal tumors and (3) Other tumors including melanocytic tumors, vascular tumors, neural tumors and others (Xanthelasma, Xanthagranuloma, etc.).

The study was approved by Institutional Ethics Committee, and research adhered to the tenets of the Declaration of Helsinki.

\section{Results}

Total number of patients who had surgery for eyelid tumor was 1502. Although the age of the patients was between 4-96 years old, most of the patients (1385 patients, $92.2 \%$ ) were above 40 years. Mean age was $59.7 \pm 17.8$ years. Male/female ratio was $697 / 805(46.4 \%$ male, $53.6 \%$ female). Eighteen patients had at least two histopathologic diagnoses because of 2 or more lesions in the eyelids; total number of the histopathologic diagnoses was 1541 .

The most frequent tumors were epidermal tumors (908 lesions, 58.9\%). Of all epidermal tumors, benign, premalignant and malignant tumors comprised $87.2 \%$, $10.4 \%$ and $2.4 \%$, respectively. Among benign epidermal tumors, squamous papilloma was the most frequent diagnosis. Squamous papilloma alone comprised $42.4 \%$ of benign epidermal lesions, $37.0 \%$ of all epidermal tumors and $21.8 \%$ of all eyelid lesions. Clinical diagnosis was a wart in 314 over 336 squamous papilloma cases $(93.5 \%)$. Five patients with basal cell carcinoma, two patients with xanthelasma, 4 patients with chalazion were misdiagnosed as squamous papilloma.

Other frequent benign epidermal tumors were seborrheic keratosis, epidermal cyst and follicular keratosis. One patient with basal cell carcinoma, five patients with melanocytic nevi and four patients with actinic keratosis were misdiagnosed as seborrheic keratosis. Seborrheic keratosis was the most frequent tumor among patients above 60 years-old $(38.6 \%$ of epidermal lesions and $24.7 \%$ of all lesions). Other misdiagnoses for seborrheic keratosis were basal cell carcinoma in 1 patient, actinic keratosis in 12 patients, melanocytic nevus in 5 patients.

Basal cell carcinoma accounted for $95.5 \%$ of epidermal malignant tumors (21 of 22 tumors). Seventeen of 21 basal cell carcinoma (80.9\%) was on the lower eyelid. Two patients had two basal cell carcinoma lesions. Surgical borders of the first resection were non-invaded in 18 of 21 tumors. The single patient with squamous cell carcinoma ( $0.1 \%$ of all epidermal tumors) had a history of rapid tumor growth. This patient was prediagnosed clinically as keratoacanthoma and histopathologically diagnosed as

Table 1. The Distribution of Epidermal Tumors (n=908)

\begin{tabular}{|c|c|c|c|c|}
\hline Type of Tumor & $\begin{array}{l}\text { umber of patients } \\
\text { ong epidermal tumors) }\end{array}$ & $\%$ among all tumors & Age (min-max) & Gender $(\mathrm{F} / \mathrm{M})$ \\
\hline \multicolumn{5}{|l|}{ Benign tumors } \\
\hline Squamous papilloma & $336(37.0)$ & 21.8 & $59(8-82)$ & $183 / 153$ \\
\hline Seborrheic keratosis & $266(29.3)$ & 17.3 & $67(19-92)$ & $154 / 112$ \\
\hline Epidermal cyst & $111(12.2)$ & 7.2 & $50(4-74)$ & $68 / 43$ \\
\hline Inverted follicular keratosis & $35 \quad(3.8)$ & 2.3 & $58(18-81)$ & $20 / 15$ \\
\hline Keratoacanthoma & $34 \quad(3.7)$ & 2.2 & $53(5-79)$ & $24 / 10$ \\
\hline Dermoid cyst & $10(1.1)$ & 0.6 & $28(4-59)$ & $4 / 6$ \\
\hline \multicolumn{5}{|l|}{ Premalign lesions } \\
\hline Actinic keratosis & $90 \quad(9.9)$ & 5.8 & $68(48-90)$ & $62 / 28$ \\
\hline Bowen disease (carcinoma in situ) & $4 \quad(0.4)$ & 0.3 & $71(69-72)$ & $2 / 2$ \\
\hline \multicolumn{5}{|l|}{ Malign tumors } \\
\hline Basal cell carcinoma & 21 & 1.4 & $69(39-79)$ & $15 / 6$ \\
\hline Squamous cell carcinoma & $1(0.1)$ & 0.06 & 76 & $0 / 1$ \\
\hline
\end{tabular}
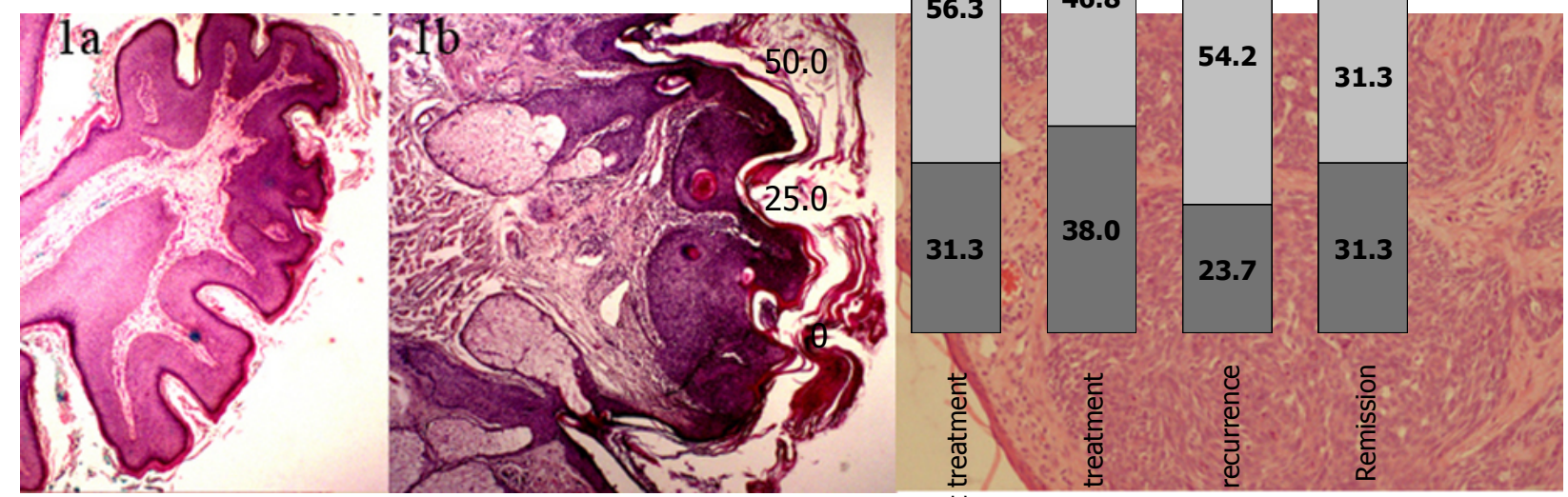

Figure 1. Examples of Histopathologic Views of Epidermal Tumors. 1a. Squamous papilloma, finger-like projections of thickened acanthotic epidermis overlying a fibrovascular core (HE x 40). 1b. Seborrheic keratosis, expansile platelike growth of the epidermis with expanded and anastomosing rete ridges (HE x 40). 1c. Basal cell carcinoma, dermal islands of the tumor cells which resemble basal keratinocytes (HE x 40) 
squamous cell carcinoma. Table 1 shows the frequency of histological subtypes and the age and sex ratio of patients with epidermal tumors. Figure 1 shows histopathologic views of some epidermal tumors.

Two hundred and two (13.1\%) tumors had adnexal origin. The most common tumors were originated from eccrine and apocrine glands and comprised $83.7 \%$ of adnexal tumors. Sebaceous gland tumors and hair follicle tumors comprised $12.9 \%$ and $0.4 \%$ of adnexal tumors, respectively. None of the patients in this study had sebaceous cell carcinoma. Table 2 summarizes the frequencies and age- and gender characteristics of adnexal tumors. Figure 2 shows histopathologic views of a trichofolliculoma and a tichoadenoma.

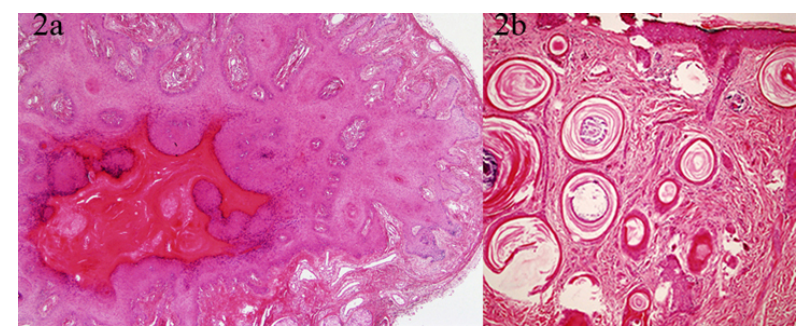

Figure 2. Examples of Histopathologic Views of Adnexal Tumors. 2a. Trichofolliculoma, dilated hair follicle lined by stratified squamous epithelium (HE x 40). 2b. Trichoadenoma, small horn cysts filled with keratin are present within the basaloid epithelial nests surrounded by dense fibroblastic stroma (HE x 40)

Table 2. The Distribution of Adnexal Tumors $(n=202)$
The tumors other than epidermal and adnexal origin comprised $27.9 \%$ of all tumors. This group included melanocytic tumors, vascular tumors, tumors of neural crest, and other tumors such as the xanthelasma, xanthagranuloma, fibrous histiocytoma, lipoma and metastatic tumors. Melanocytic lesions were the most common among these tumors $(64.7 \%)$ and also second most common group of eyelid tumor after squamous papilloma. Xanthelasma comprised $26.9 \%$ of this group of tumors. However, vascular tumors comprised only $4.1 \%$. Table 3 summarizes the frequencies and age- and gender characteristics of 'other' tumors. Figure 3 shows histopathologic views of a cavernous hemangioma and a neurofibroma.

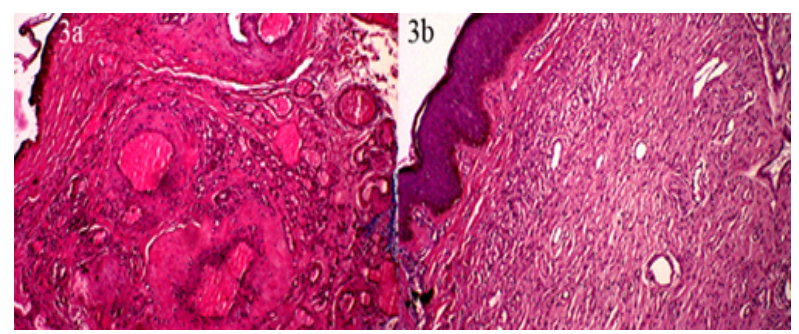

Figure 3. Examples of Histopathologic Views of Other Tumors. 3a. Cavernous hemangioma, cavernous vascular spaces separated by connective tissue in the dermis (HE x 40). $3 \mathrm{~b}$. Neurofibroma, tortuous proliferation of all components of peripheral nerves including axons, Schwann cells, fibroblasts and perineurial cells $(\mathrm{HE}, \mathrm{x} 40)$

\begin{tabular}{lcccr}
\hline Type of Tumor & $\begin{array}{c}\text { Number of patients } \\
\text { among epidermal tumors) }\end{array}$ & $\%$ among all tumors & Age (min-max) & Gender (F/M) \\
\hline $\begin{array}{l}\text { Sebaceous gland tumors } \\
\text { Sebaceous adenoma }\end{array}$ & $18(8.9)$ & 1.1 & $64(51-78)$ & $8 / 10$ \\
Sebaceous hyperplasia & $8(3.9)$ & 0.5 & $62(49-73)$ & $2 / 1$ \\
Tumors of the eccrine and apocrine gland origin & $164(81.1)$ & 10.6 & $55(28-76)$ & $75 / 89$ \\
Hydrocystoma & $5(2.4 \%)$ & 0.3 & $51(20-74)$ & $3 / 2$ \\
Syringoma & $3(1.5 \%)$ & 0.2 & $69(62-75)$ & $1 / 2$ \\
Hair follicle tumors & $2(1)$ & 0.13 & $51(50-52)$ & $2 / 0$ \\
Trichilemmoma & $1(0.5)$ & 0.06 & 61 & $1 / 0$ \\
Trichoepithelioma & $1(0.5)$ & 0.06 & 58 & $0 / 1$ \\
Trichofolliculoma & & & & \\
Trichoadenoma & & & & \\
\hline
\end{tabular}

Table 3. The Distribution of Other Tumors $(n=431)$

\begin{tabular}{|c|c|c|c|c|}
\hline Type of Tumor & $\begin{array}{l}\text { Number of patients } \\
\text { (\% among tumors) }\end{array}$ & $\begin{array}{l}\% \text { among } \\
\text { all tumors }\end{array}$ & Age (min-max) & Gender $(\mathrm{F} / \mathrm{M})$ \\
\hline \multicolumn{5}{|l|}{ Melanocytic tumors } \\
\hline Nevus & $272(63.1)$ & 17.6 & $56(4-83)$ & $151 / 121$ \\
\hline Specific subtypes (kissing nevus, b & us, etc) $7(1.6)$ & 0.5 & $42(4-60)$ & $5 / 2$ \\
\hline \multicolumn{5}{|l|}{ Vascular tumors } \\
\hline Capillary hemangioma & $14(3.2)$ & 0.9 & $5(3-10)$ & $10 / 4$ \\
\hline Cavernous hemangioma & $4(0.9)$ & 0.3 & $57(42-63)$ & $2 / 2$ \\
\hline \multicolumn{5}{|l|}{ Tumors of neural crest origin } \\
\hline Plexiform Neurofibroma & $5(1.1)$ & 0.3 & $42(11-59)$ & $1 / 4$ \\
\hline Solitary circumscribed neurinoma & $3(0.7)$ & 0.2 & $52(45-66)$ & $1 / 3$ \\
\hline Traumatic neuroma & $2(0.4)$ & 0.13 & $46(41-51)$ & $0 / 2$ \\
\hline \multicolumn{5}{|l|}{ Other } \\
\hline Xanthelasma & $116(26.9)$ & 7.6 & $52(21-73)$ & $69 / 37$ \\
\hline Xanthagranuloma & $2(0.4)$ & 0.13 & $6(4-8)$ & $1 / 1$ \\
\hline Fibrous histiocytoma & $1(0.2)$ & 0.06 & 56 & $0 / 1$ \\
\hline Lipoma & $4(0.9)$ & 0.3 & $52(39-65)$ & $1 / 3$ \\
\hline Metastatic tumors & $1(0.2)$ & 0.06 & 61 & $1 / 0$ \\
\hline
\end{tabular}




\section{Discussion}

To the best of our knowledge, this study is the largest histopathological cohort of eyelid tumors reported to date from Turkey. Because of the recent (after 2005) significant improvements in health care, and social security system in Turkey, people began to gain easy access to medical centers. This change let medical centers examine a large number of patients in a relatively short period. The authors had been able to see 1502 patients with eyelid tumor in the last three years. This study did not include patients with eyelid tumors who applied to dermatologists and plastic surgeons. However, the large number of patients included in this study gives a remarkable knowledge about the distributions of eyelid tumors in Ankara, Turkey.

The largest study reported for the frequencies of eyelid tumors was published by Deprez et al.(Deprez and Uffer, 2009) The authors reported the clinical and histopathological features of 5504 eyelid skin tumors. Although the number of the patients included in this study is almost one fourth of the study performed by Deprez et al, we think that it may give more reliable epidemiologic data than Deprez et al.'s study. Deprez et al. reported the data of patients half of whom were referred from 20 private ophthalmologists' offices. Referral of the patients may have caused selection bias. However, in this study, all the histopathologic data were collected from the first ophthalmology center that the patients applied. In addition, almost all the eyelid tumoral lesions examined were surgically removed. This makes the result of this study epidemiologically more remarkable.

Deprez et al.(Deprez and Uffer, 2009) reported that five most frequent subtypes of eyelid tumors were squamous cell papilloma (26\%), seborrheic keratosis, melanocytic nevus (20\%), hydrocystoma (8\%), and xanthoma/xanthelasma (6\%). In that report, benign tumors represented $84 \%$ of the cases. In our study, 5 most common tumors were squamous papilloma, melanocytic nevus, seborrheic keratosis, epidermal cyst and hydrocystoma. In this study, benign lesions comprised $87.2 \%$ of all lesions. One other retrospective analysis of eyelid tumors was accomplished by Coroi et al.(Coroi et al., 2010) The authors reported that there were 114 pseudotumors, 102 benign tumors and 225 cases of malignant tumors among in-patients of their hospital in the last eight years.

Recently Bagheri et al. reported the 10-year analyses of eyelid masses in a third referral center in Tehran.(Bagheri et al., 2013) The authors reported that $6 \%$ of malignant tumors were sebaceous cell carcinoma. It should be emphasized that the high rate of sebaceous cell carcinoma in that study is possibly because of the selection bias because of the center that the study conducted; that is a third-referral center. However, our study was performed in an eye center that could be easily reached by the patients. Almost all eyelid masses were excised. This has possibly caused low rate of malignancies in this study.

Different frequencies were reported about the eyelid malignancies in the literature with basal cell carcinoma as the most frequent malignancy.(Hilovsky, 1995; Chang et al., 2003; Takamura and Yamashita, 2005; Lin et al.,
2006; Coroi et al., 2010) Male dominance was seen in basal cell carcinoma in accordance with previous findings (Frost et al., 1998). Basal cell carcinoma is most common in the lower eyelid, and medial canthal area in the ocular adnexal region (Margo and Waltz, 1993; Allali et al., 2005; Mannor et al., 2009). This tumor generally does not metastasize, however it is locally invasive, and destructive. In this study, 13 of 16 cases had nodular-ulcerative disease, 2 patients had morpheaform or sclerosing subtype and 1 patient had baso-squamous subtype of basal cell carcinoma. Orbital invasion was not observed in any case with basal cell carcinoma. Squamous cell carcinoma is the second most common form of eyelid cancer and represents less than 5\% of all eyelid malignancies.(Piest, 1992) In this study, only 1 case of squamous cell carcinoma was diagnosed $(4.5 \%)$. Sebaceous cell carcinoma is a rare eyelid tumor and originates from folliculo-sebaceous apocrine unit. It is more common in the upper eyelidmaybe because of the more meibomian glands in the upper eyelid. Sebaceous cell carcinoma is known as a masquerader as it may mimic benign eyelid conditions such as chronic blepharoconjunctivitis or chalazion and may be misdiagnosed for months, or even years (Lai et al., 2004). In this study, it was surprising for us that we did not diagnose any patient with sebaceous cell carcinoma. Metastases to the eyelids are rare, accounting for less than $1 \%$ of all malignant eyelid tumors. Melanoma and breast cancer are the most common cancers that metastasize to the eyelids.(Bianciotto et al., 2009) In this study too, we diagnosed only one patient with eyelid metastasis that the primary tumor was in the breast. No specific treatment was applied for eyelid lesion.

Squamous papilloma is the most common benign eyelid lesion and is defined by ophthalmopathologists as 'fingerlike projections of vascularized connective tissue covered by acanthotic epithelium with focal hyperkeratosis and parakeratosis (Deprez and Uffer, 2009). These lesions are flesh colored and may be sessile or pedunculated. Squamous papilloma is also called fibroepithelial polyp, acrochordon and skin tag. The second most common lesion in this study was seborrheic keratosis. In this study, almost one sixth of patients with seborrheic keratosis were misdiagnosed as nevus, pigmented basal cell carcinoma, actinic keratosis, and squamous papilloma.

Actinic keratosis arises on fair-skinned people in areas of long-term sun exposure.(Salasche, 2000) Long-term ultraviolet light exposure is implicated as the cause from both epidemiologic observations and molecular analysis of tumor cells (Leffell, 2000; Salasche, 2000). Similar to previous findings,(Salasche, 2000) in this study, actinic keratosis was approximately 2 times more frequent in men than in women ( 62 male, 28 female). It is thought to be related to the higher incidence of outdoor occupation of men than women and hence a greater cumulative ultraviolet exposure (Frost et al., 1998).

This is the largest epidemiological data for eyelid tumors from Turkey. The histopathologic diagnosis increases the accuracy of the data and the value of this study. The absence of the sebaceous carcinoma in the large number of patients examined deserves to be stressed. 


\section{Acknowledgements}

The authors of this study did not receive any financial support for this submission. All of the authors declare that there is no conflict of interest for this submission.

\section{References}

Abdi U, Tyagi N, Maheshwari V, et al (1996). Tumours of eyelid: a clinicopathologic study. J Indian Med Assoc, 94, 405-9.

Allali J, D’Hermies F, Renard G (2005). Basal cell carcinomas of the eyelids. Ophthalmologica, 219, 57-71.

Bagheri A, Tavakoli M, Kanaani A, et al (2013). Eyelid masses: a 10 -year survey from a tertiary eye hospital in Tehran. Middle East Afr J Ophthalmol, 20, 187-92.

Bhurgri Y, Bhurgri H, Usman A, et al (2003). Epidemiology of ocular malignancies in Karachi. Asian Pac J Cancer Prev, 4, 352-7.

Bianciotto C, Demirci H, Shields CL, et al (2009). Metastatic tumors to the eyelid: report of 20 cases and review of the literature. Arch Ophthalmol, 127, 999-1005.

Chang CH, Chang SM, Lai YH, et al (2003). Eyelid tumors in southern Taiwan: a 5-year survey from a medical university. Kaohsiung $J$ Med Sci, 19, 549-54.

Coroi MC, Rosca E, Mutiu G, et al (2010). Eyelid tumors: histopathological and clinical study performed in County Hospital of Oradea between 2000-2007. Rom J Morphol Embryol, 51, 111-5.

Deprez M, Uffer S (2009). Clinicopathological features of eyelid skin tumors. A retrospective study of 5504 cases and review of literature. Am J Dermatopathol, 31, 256-62.

Frost CA, Green AC, Williams GM (1998). The prevalence and determinants of solar keratoses at a subtropical latitude (Queensland, Australia). Br J Dermatol, 139, 1033-9.

Gajda M, Kaminska-Winciorek G (2014). Do not let to be late: overview of reasons for melanoma delayed diagnosis. Asian Pac J Cancer Prev, 15, 3873-7.

Hilovsky JP (1995). Lid lesions suspected of malignancy. J Am Optom Assoc, 66, 510-5.

Lai TF, Huilgol SC, Selva D, et al (2004). Eyelid sebaceous carcinoma masquerading as in situ squamous cell carcinoma. Dermatol Surg, 30, 222-5.

Leffell DJ (2000). The scientific basis of skin cancer. J Am Acad Dermatol, 42, 18-22.

Lin HY, Cheng CY, Hsu WM, et al (2006). Incidence of eyelid cancers in Taiwan: a 21-year review. Ophthalmology, 113, 2101-7.

Mannor GE, Chern PL, Barnette D (2009). Eyelid and periorbital skin basal cell carcinoma: oculoplastic management and surgery. Int Ophthalmol Clin, 49, 1-16.

Margo CE, Waltz K (1993). Basal cell carcinoma of the eyelid and periocular skin. Surv Ophthalmol, 38, 169-92.

Ni Z (1996). [Histopathological classification of 3,510 cases with eyelid tumor]. Zhonghua Yan Ke Za Zhi, 32, 435-7.

Piest KL (1992). Malignant lesions of the eyelids. J Dermatol Surg Oncol, 18, 1056-9.

Salasche SJ (2000). Epidemiology of actinic keratoses and squamous cell carcinoma. J Am Acad Dermatol, 42, 4-7.

Salomon J, Bieniek A, Baran E, et al (2004). Basal cell carcinoma on the eyelids: own experience. Dermatol Surg, 30, 257-63.

Takamura H, Yamashita H (2005). Clinicopathological analysis of malignant eyelid tumor cases at Yamagata university hospital: statistical comparison of tumor incidence in Japan and in other countries. Jpn J Ophthalmol, 49, 349-54.

Turgay AS, Sari D, Can M, et al (2005). Determination of sunburn and skin cancer risk of outpatients in a dermatology polyclinic. Asian Pac J Cancer Prev, 6, 143-6.
$\mathrm{Xu}$ XL, Li B, Sun XL, et al (2008). Clinical and pathological analysis of 2639 cases of eyelid tumors. Zhonghua Yan Ke Za Zhi, 44, 38-41 (in Chinese). 